\title{
ON THE POSITIVE SPECTRUM OF SCHRÖDINGER OPERATORS WITH LONG RANGE POTENTIALS
}

BY

\author{
G. B. KHOSROVSHAHI ${ }^{1}$, H. A. LEVINE AND L. E. PAYNE ${ }^{2}$
}

\begin{abstract}
In this paper we are concerned with solutions of the equation $\Delta u+p(x) u=0$ in an unbounded domain $\Omega$ in $\mathbf{R}^{n}, \Omega \supset\left\{x \mid\|x\|>R_{0}\right\}$. The main result is a determination of conditions on the asymptotic behavior of $p(x)$ sufficient to guarantee that no nontrivial $L_{2}$ solution exists. Our results contain those of previous authors as special cases. The principal application is to the determination of upper bounds for positive eigenvalues of Schrödinger operators.
\end{abstract}

0. Introduction. One of the most important questions in the analytic theory of quantum mechanics is the question of existence of eigenvalues of Hamiltonians (bound states). It is intriguing, in particular, to investigate the question of existence of positive eigenvalues of Hamiltonians of Schrödinger type. For such problems physical intuition suggests a negative answer, but mathematically the question is a very subtle one which requires rigorous investigation.

In this paper we will denote by $H$ any selfadjoint realization of $-\Delta+V$ in which $\Delta$ is the $n$-dimensional Laplacian $(n \geqslant 3)$, and $V$ is a real valued function defined over the $n$-dimensional euclidean space $\mathbf{R}^{n}$ (where $x=$ $\left.\left(x_{1}, \ldots, x_{n}\right), r=\|x\|\right)$. The set $\Omega$ is an unbounded open connected domain in $\mathbf{R}^{n}$ such that $\Omega \supset\left\{x \mid\|x\| \geqslant R_{0}\right\}$ for some $R_{0} \in \mathbf{R}$. In general, it is believed that the spectrum of $H$ consists of $[0, \infty) \cup\left\{\lambda_{j}\right\}$ where $[0, \infty)$ constitutes the continuous spectrum of the operator, and $\lambda_{i}$ 's are discrete negative eigenvalues with finite multiplicity. Since the essential spectrum of the operator $H$ is more stable under certain classes of perturbations than the continuous spectrum it is desirable to show that the continuous spectrum coincides with the essential spectrum, and for many operators this is the case. There is, however, a celebrated example due to von Neumann and Wigner [11] in which, for a reasonable potential, the operator $-\Delta+V$ possesses a positive eigenvalue. This example suggests the possibility that for certain

\footnotetext{
Received by the editors February 27, 1978.

AMS (MOS) subject classifications (1970). Primary 35J10, 47A40.

${ }^{1}$ The research of the first author was supported in part by the Iranian National Council grant while the author was on sabbatical leave at Cornell University.

${ }^{2}$ The research of the second and third authors was supported in part by NSF grant MCS-77-01273.
} 
classes of operators with oscillating long range potentials there may exist positive eigenvalues immersed in the continuous spectrum.

The plan of the paper is as follows: We first give a reasonably thorough account of the literature on nonexistence of positive eigenvalues. We then state our main result, which contains as special cases many of the known results and in addition applies to a much wider class of potentials. We then prove our main theorem and give some examples.

(1) Kato [4] has shown that for one body problems with $V(x)=O\left(r^{-\alpha}\right)$, as $r \rightarrow \infty$ for $\alpha>1$, there are no positive eigenvalues.

(2) It follows, in fact, from Kato's Theorem 1.a [4] that if for some constant $K>0,|r V(x)| \leqslant K$ for $r \geqslant R_{0}$, then there exists no positive eigenvalue $\lambda$ satisfying $\lambda>K^{2}$.

(3) In [9], Odeh has shown that if $V(x)=O(1)$ as $r \rightarrow \infty$, and $\partial V / \partial r<0$ for large $r$, then $H$ has no positive eigenvalues.

(4) In [12], Weidmann has proved that if $V(x)$ is a homogeneous function of degree $-\alpha, 0<\alpha<1$, i.e.,

$$
r \partial V(x) / \partial r+\alpha V(x) \leqslant 0 \text { for } x \in M=\{x|| V(x) \mid<\infty\},
$$

then the spectrum of $H$ is purely continuous.

(5) Finally, Simon has obtained the following theorem [10]:

THEOREM 1. If $V$ is a real valued function in $\mathbf{R}^{3}$ with the properties that

(i) $V \in L_{2}\left(\mathbf{R}^{3}\right)+L_{\infty}\left(\mathbf{R}^{3}\right)$; and $V=V_{0}+V_{1}$ where $V_{0}$ and $V_{1}$ are $C^{\infty}$ in $\left\{x \mid\|x\| \geqslant R_{0}\right\}$ for some $R_{0}$,

(ii) $V_{0}(x)=o(1)$ as $r \rightarrow \infty$,

$$
\limsup _{r \rightarrow \infty} r \partial V_{0}(x) / \partial r=\Lambda_{0}<\infty,
$$

while $V_{1}(x)=o\left(r^{-1}\right)$ as $r \rightarrow \infty$,

(iii) solutions of $\mathrm{Hu}=\lambda u$ satisfy the unique continuation property, then $H$ has no eigenvalues in $\left(\Lambda_{0}, \infty\right)$.

REMARKS. In (3), (4), and (5) the authors have employed a variant of Kato's method [4] and have assumed some degree of smoothness for a part of the potential $V$. Also, it is worth noting that in (4) and (5) results were established for the special case $\Omega=\mathbf{R}^{n}$ and $\mathbf{R}^{3}$ respectively.

(6) Weidmann in [13] obtained a virial theorem which asserts that under appropriate conditions on $V$ it follows that $\langle[r \partial V / \partial r+2(V-\lambda)] u, u\rangle=0$, where $\lambda$ is an eigenvalue and $u$ is the corresponding eigenfunction of $H$. This, in part, implies that if

$$
r \frac{\partial V(x)}{\partial r}+\gamma V(x) \leqslant 0 \quad(0<\gamma \leqslant 2, x \neq 0),
$$

then $H$ has no eigenvalues in $[0, \infty)$. In general, virial theorems are state- 
ments about equipartitioning of the energy of the related systems.

(7) The following result is due to Agmon [1, Theorem 4]:

THEOREM 2. Suppose that $V(x)$ is a real function, $V \in L_{2}^{\text {loc }}(\Omega)$ and satisfies the following conditions:

(i) $V(x)$ is locally Hölder continuous in a connected open set $\Omega_{0} \subset \Omega$ where $\Omega_{0} \supset\left\{x \mid\|x\| \geqslant R_{0}\right\}$ and $\Omega-\Omega_{0}$ is a set of measure zero.

(ii) For $r \geqslant R_{0}$

$$
V(x)=V_{0}(x)+V_{1}(x)
$$

where $V_{0}$ is a real continuous function possessing a continuous radial derivative satisfying

$$
V_{0}(x)=o(1), \quad \limsup _{r \rightarrow \infty} r \frac{\partial V_{0}}{\partial r}=\Lambda_{0}
$$

while

$$
V_{1}(x)=O\left(r^{-1-\varepsilon}\right), \quad r \rightarrow \infty \text { for some } \varepsilon>0 .
$$

Under these conditions, $H$ as an operator in $L_{2}(\Omega)$ has no eigenvalues $\lambda>\Lambda_{0} / 2$.

(8) Khosrovshahi, in [6], proved a slightly different version of Agmon's theorem.

THEOREM 3. In Theorem 2 , if we replace (**) by

$$
\underset{r \rightarrow \infty}{\lim \sup }\left|r V_{1}(x)\right| \leqslant K
$$

then $H$ has no eigenvalues $\lambda>\left(K+\sqrt{K^{2}+\Lambda_{0} / 2}\right)^{2}$.

REMARKs. (1) Agmon has improved Simon's result by a factor of 1/2. (2) Theorem 3 can handle a wider range of potentials than those of Agmon. In fact, by Theorem 3, one obtains Agmon's result if $K$ is equal to zero. On the other hand, if $\Lambda_{0}=0$, Theorem 3 fails to yield Kato's result, namely that there exists no $\lambda$ satisfying $\lambda>K^{2}$. Thus Theorem 3 is a combination of Agmon and Kato type results but not quite in the optimal sense. For the von Neumann potential $V(r)=-8 \sin 2 r / r+O\left(r^{-2}\right)$ we are assured, by Theorem 1 , that there is no eigenvalue $\lambda>16$, whereas, by Theorems 2 and 3 , there can exist no $\lambda>8$. (3) Kato, in [4], has introduced a class of von Neumann type potentials, and the conclusions of Theorems 1, 2, and 3 can be applied to these potentials as well.

From the above cited results, it is clear that the class of long range potentials for which one can establish the nonexistence of positive eigenvalues is rather restrictive. Our work extends the size of this class considerably.

(9) In this paper we obtain the following theorem: 
THEOREM 4. Let $V(x) \in L_{2}^{\text {loc }}(\Omega)$ be a real function satisfying hypothesis (i) of Theorem 2 and suppose it is possible to choose an $R_{0}$ sufficiently large such that for $\|x\| \geqslant R_{0}$

(ii) $V(x)=V_{0}(x)+V_{1}(x)+V_{2}(x)$

where $V_{0}(x)$ is real, continuous, possesses a continuous radial derivative and satisfies (*), $V_{1}$ is real and satisfies $(* * *)$, and $V_{2}(x)$ is such that (representing $V_{2}(x)$ is spherical coordinates as $\left.V_{2}(r, \omega)\right)$

$$
\sup _{\omega}\left|\int_{r}^{\rho} \sigma V_{2}(\sigma, \omega) d \sigma\right| \leqslant M<\frac{1}{4} \text { for all } \rho, r \geqslant R_{0} \text {. }
$$

If we set

$$
\alpha \equiv \max \left\{\frac{\left[K+\sqrt{K^{2}+2 \Lambda_{0}(1-2 M)^{2}}\right]^{2}}{4(1-2 M)^{2}}, \frac{2 K^{2}+\Lambda_{0}(1-4 M)}{2(1-4 M)^{2}}\right\},
$$

then the unique selfadjoint extension operator $H$ possesses no eigenvalues in $(\alpha, \infty)$.

REMARKS. (1) As Lavine [8] has noted, although wave operators do not exist for the Coulomb potential $(V(x)=-c / r)$, the theory of long range potentials is not yet complete. The problem, from the physicist's viewpoint, is that scattering theory without long range potentials cannot be considered satisfactory. The $V_{1}$ of Theorem 4 is such a long range potential. (2) The $V_{2}$ piece of $V$ makes it possible to include some unbounded potentials which oscillate through arbitrarily large positive and negative values as $r \rightarrow \infty$ but which according to Kato [5] do not violate the conditions for uniqueness of the selfadjoint extension of the operator $H$. (See [5].) (3) We note that if $V \equiv V_{0}$ (i.e., $K=0, M=0$ ) we retrieve Agmon's result (Theorem 2), whereas if $V \equiv V_{1}$ (i.e., $\Lambda_{0}=0, M=0$ ) we retrieve Kato's result that there exist no eigenvalues $\lambda$ in the interval $\lambda>K^{2}$.

(10) Finally, we mention a result of Konno [7] derived for a region $\Omega$ with noncompact boundary. Specifically, $\Omega$ includes the exterior of a certain parabaloid of revolution, and $V$ is a real valued function, an element of $C^{1}(\Omega)$, which satisfies

$$
|V(x)|=o(1), \quad|\operatorname{grad} V(x)|=O\left(r^{-3 / 2}\right) \quad \text { as } r \rightarrow \infty .
$$

Under these conditions any function $u$ which satisfies

$$
-\Delta u+V(x) u=\lambda u, \quad u \in L_{2}(\Omega),
$$

must vanish everywhere in $\Omega$.

In the remaining part of this article, we furnish the proof of Theorem 4, and then give some examples. Theorem 4 will be a special case of the more general Main Theorem treated in the next section. 
I. Main result, nonexistence of $L_{2}$ solutions. Let $\Omega \subset \mathbf{R}^{n}$ be an unbounded connected open region such that the exterior $B^{c}\left(R_{0}\right)$ of a sphere of radius $R_{0}$ is contained in $\Omega$, i.e., $B^{c}\left(R_{0}\right) \equiv\left\{x \mid\|x\| \geqslant R_{0}\right\} \subset \Omega$, where $\|x\|=r=$ $\left(\sum_{1}^{n} x_{i}^{2}\right)^{1 / 2}$ with some suitably chosen origin. We shall determine conditions on the real potential $p(x)$ such that the equation

$$
\Delta u+p(x) u=0
$$

has no nontrivial $L_{2}$ solutions in $\Omega$. We shall be concerned throughout with classical solutions of (1.1), i.e., with solutions which belong to $W^{2, \infty}(\Omega) \cap$ $L_{2}(\Omega)$. We establish the following theorem:

MAIN THEOREM. Let $u \in W^{2, \infty}$ be a real valued solution of $(1.1)$ in $\Omega$, where $p(x) \in L_{2}^{\mathrm{loc}}(\Omega)$ is a real valued function representable in $B^{c}\left(R^{*}\right)$ as

$$
p(x)=p_{0}(x)+p_{1}(x)+p_{2}(x) ;
$$

for sufficiently large $R^{*}>R_{0}$; then if the quantities $p_{i}(x)$ satisfy the properties

(a) $\sup _{x \in B^{c}\left(R^{*}\right)}\left|r p_{1}(x)\right| \leqslant K, K$ a positive constant;

(b) $\sup _{\|\omega\|=1}\left|\int_{r}^{\rho} \sigma p_{2}(\sigma \omega) d \sigma\right| \leqslant M<\frac{1}{4}, \forall \rho, r \geqslant R^{*}$,

(c) $p_{0}(x)$ is a real continuous function with a continuous radial derivative and $p_{0}(x) \geqslant K_{1}>0$;

(d) $r \partial p_{0}(x) / \partial r+\left(2-\hat{\gamma}-4 M-\varepsilon_{1}\right) p_{0}(x)-K^{2} \hat{\gamma}^{-1} \geqslant \varepsilon_{2}$, for some $\hat{\gamma}>0$;

(e) $r \partial p_{0}(x) / \partial r+2\left(1-4 M-\varepsilon_{3}\right) p_{0}-2 K^{2}\left(1-4 M-\varepsilon_{4}\right)^{-1} \geqslant \varepsilon_{5}$;

where the $\varepsilon_{i}$ 's can be made arbitrarily small by choosing $R^{*}$ sufficiently large, it follows that any solution $u \in L_{2}\left(B^{c}\left(R^{*}\right)\right)$ must vanish identically in $B^{c}\left(R^{*}\right)$. If in addition $p(x)$ is Hölder continuous in a connected open set $\Omega_{0} \subset \Omega$ where $\operatorname{meas}\left(\Omega \mid \Omega_{0}\right)=0$ then $u \equiv 0$ in $\Omega$.

Before giving a proof of the Main Theorem we indicate how Theorem 4 follows from the Main Theorem.

Proof of Theorem 4. Assume $H$ possesses an eigenvalue $\lambda \in(\alpha, \infty)$. Let $u$ be the corresponding eigenfunction such that

$$
\Delta u+(\lambda-V(x)) u=0 \text { in } \Omega \text { (distributional sense). }
$$

It follows from assumption (i) of Theorem 2 that $\left.u\right|_{\Omega_{0}}$ is a $C^{2}$ function in $\Omega_{0}$. Since meas $\left(\Omega \mid \Omega_{0}\right)=0$, it follows that $u \neq 0$ in $\Omega_{0}$.

We rewrite

$$
p(x)=\lambda-V(x)=p_{0}(x)+p_{1}(x)+p_{2}(x) \text { in } B^{c}\left(R^{*}\right),
$$

and choose $p_{0}=\lambda-V_{0}(x), p_{1}(x)=-V_{1}(x)$, and $p_{2}(x)=-V_{2}(x)$. We observe that all the conditions of the theorem are satisfied. Thus $u \equiv 0$ in $B^{c}\left(R^{*}\right)$, for $R^{*}$ sufficiently large, and by the unique continuation theorem, $u$ must vanish in $\Omega_{0}$ contrary to our assumption. It might be mentioned here that (d) and (e) yield the lower bound, $\alpha$, for the nonexistence interval in Theorem 4, if one replaces $p_{0}$ by $\lambda$ and $r \partial p_{0} / \partial r$ by $-\Lambda_{0}$ in (d) and then 
optimalizes the resulting inequality with respect to $\hat{\gamma}$.

To establish the Main Theorem we shall assume that $u \neq 0$ is an $L_{2}$ solution of (1.1) and use a series of lemmas which are consequences of conditions (a)-(e) together with a lemma derived in [6] in order to arrive at a contradiction. For completeness we include this latter lemma.

LEMMA 1 (MAIN LEMMA OF [6]). Let $F$ be a nonnegative real valued function of $t$, continuous in the half internal $\left(0, t_{0}\right]$ and twice continuously differentiable in $\left(0, t_{0}\right)$, where $t_{0}$ is an arbitrary finite number.

Suppose further that for all $t \in\left(0, t_{0}\right]$ we have

$$
\int_{0}^{t} \eta^{-\alpha_{2}} F(\eta) d \eta<\infty
$$

and that

$$
F \frac{d^{2} F}{d t^{2}}-\left(\frac{d F}{d t}\right)^{2} \geqslant-C_{1} t^{-1} F \frac{d F}{d t}+\varepsilon t^{-\alpha_{1}} F \int_{0}^{t} \eta^{-\alpha_{2}} F(\eta) d \eta
$$

where $C_{1}$ and $\varepsilon$ are strictly positive, $\alpha_{2}>1$, and $\alpha_{1}+\alpha_{2}>1+2 C_{1}$. Under these conditions

$$
F(t) \equiv 0 \quad \forall t \in[0, t]
$$

We first sketch an outline of the proof of the Main Theorem. Since we are assuming that $u \in L_{2}(\Omega)$, it follows that

$$
F(r) \equiv \int_{r}^{\infty} \oint_{S_{\rho}} \rho^{-2(n-1)} u^{2} d s d \rho \quad\left(r \geqslant R_{0}\right)
$$

is well defined. We shall view $F$ as a function of $t=r^{-(n-2)}$ and show that it satisfies the hypotheses of Lemma 1 . From Lemma 1 , it will follow that $u \equiv 0$ in $B^{c}\left(R^{*}\right)$. As a consequence of unique continuation theorems for elliptic equations [3], we can then conclude that $u \equiv 0$ in $\Omega$.

In order to carry out this program, we first calculate $F F^{\prime \prime}-\left(F^{\prime}\right)^{2}\left({ }^{\prime} \equiv\right.$ $\left.d / d t, t=r^{-(n-2)}\right)$. To facilitate this calculation, we recall from [0] the following consequences of the fact that $u \in L_{2}(\Omega)$ and satisfies (1.1):

$$
\begin{array}{r}
\liminf _{r \rightarrow \infty} r \oint_{S_{r}} p_{0} u^{2} d s=0 \\
\liminf _{r \rightarrow \infty} r \oint_{S_{r}}|\operatorname{grad} u|^{2} d s=0 \\
\liminf _{r \rightarrow \infty}\left|r \oint_{S_{r}} u \frac{\partial u}{\partial r} d s\right|<0
\end{array}
$$


Since $r^{n-1} d / d r=-(n-2) d / d t$, we have

$$
\begin{aligned}
-(n-2) \frac{d F}{d t} & =-\oint_{S_{r}} r^{-(n-1)} u^{2} d s \\
& =2 \int_{r}^{\infty} \oint_{S_{\rho}} \rho^{-(n-1)} u \frac{\partial u}{\partial \rho} d s d \rho-\lim _{R_{m} \rightarrow \infty} \frac{1}{R_{m}^{n-1}} \oint_{S_{R_{m}}} u^{2} d s \\
& =2 \int_{r}^{\infty} \oint_{S_{\rho}} \rho^{-(n-1)} u \frac{\partial u}{\partial \rho} d s d \rho
\end{aligned}
$$

where we have taken a sequence $R_{m} \rightarrow \infty$ in such a way that lim inf can be replaced by lim in the first of equations (A) and the limit on the right can be evaluated as zero. Similarly, using the remaining conditions (A) we obtain

$$
(n-2)^{2} F^{\prime \prime}=2 \oint_{S_{r}} u \frac{\partial u}{\partial r} d s=2 \int_{r}^{\infty} \oint_{S_{p}}\left[|\operatorname{grad} u|^{2}-p u^{2}\right] d s d \rho
$$

where we have used (1.1).

We conclude, after a routine calculation employing Schwarz's inequality, that

$$
F F^{\prime \prime}-\left(F^{\prime}\right)^{2} \geqslant 2 \frac{F(t)}{(n-2)^{2}} \int_{r}^{\infty} \oint_{S_{\rho}}\left[|\operatorname{grad} u|^{2}-p u^{2}-2\left(\frac{\partial u}{\partial \rho}\right)^{2}\right] d s d \rho
$$

We now let

$$
I_{1} \equiv \int_{r}^{\infty} \oint_{S_{p}}\left[|\operatorname{grad} u|^{2}-p u^{2}-2\left(\frac{\partial u}{\partial \rho}\right)^{2}\right] d s d \rho .
$$

Our task will be to determine an appropriate lower bound for $I_{1}$ so that when this bound is inserted into (1.4) the resulting inequality will be of the form indicated in Lemma 1.

In deriving this bound we make use of a number of integral inequalities involving $p_{1}$ and $p_{2}$. We list them here and relegate the derivations to the appendix. In the following computation we will denote $\partial u / \partial \rho$ by $u_{\rho}$ for simplicity.

$$
\begin{gathered}
\left|\int_{r}^{R} \oint_{S_{\rho}} p_{1} u^{2} d s d \rho\right| \leqslant \varepsilon \int_{r}^{R} \oint_{S_{\rho}} u^{2} d s d \rho \\
\left|\int_{r}^{R} \oint_{S_{\rho}} p_{1} u^{2} d s d \rho\right| \leqslant K \ln \left(\frac{R}{r}\right) \oint_{S_{R}} u^{2} d s \\
+\int_{r}^{R} \frac{1}{\rho} \int_{\rho}^{R} \oint_{S_{\sigma}}\left[\gamma u_{\sigma}^{2}+\frac{K^{2}}{\gamma} u^{2}\right] d s d \sigma d \rho
\end{gathered}
$$




$$
\begin{aligned}
&\left|\int_{r}^{R} \oint_{S_{\rho}} \rho p_{1} u u_{\rho} d s d \rho\right| \frac{\gamma}{2} \int_{r}^{R} \oint_{S_{\rho}}\left(u_{\rho}\right)^{2} d s d \rho+\frac{K^{2}}{2 \gamma} \int_{r}^{R} \oint_{S_{\rho}} u^{2} d s d \rho, \\
&\left|\int_{r}^{R} \frac{1}{\rho} \int_{\rho}^{R} \oint_{S_{o}} \sigma p_{1} u u_{\sigma} d s d \sigma d \rho\right| \leqslant \frac{\gamma}{2} \int_{r}^{R} \frac{1}{\rho} \int_{\rho}^{R} \oint_{S_{o}}\left(u_{\sigma}\right)^{2} d s d \sigma d \rho \\
&+\frac{K^{2}}{2 \gamma} \int_{r}^{R} \frac{1}{\rho} \int_{\rho}^{R} \oint_{S_{\sigma}} u^{2} d s d \sigma d \rho, \\
&\left|\int_{r}^{R} \oint_{S_{\rho}} p_{2} u^{2} d s d \rho\right| \leqslant \frac{M}{R} \oint_{S_{R}} u^{2} d s+\varepsilon \int_{r}^{R} \oint_{S_{\rho}}\left[u_{\rho}^{2}+u^{2}\right] d s d \rho, \\
&\left|\int_{r}^{R} \oint_{S_{\rho}} p_{2} u^{2} d s d \rho\right| \leqslant \varepsilon \int_{S_{R}} u^{2} d s+\hat{C} \ln \left(\frac{R}{r}\right) \oint_{S_{R}}\left|u u_{R}\right| d s \\
&+\int_{r}^{R} \frac{1}{\rho} \int_{\rho}^{R} \oint_{S_{\sigma}}\left(2 M p_{0}+\varepsilon\right) u^{2} d s d \sigma d \rho \\
&+(2 M+\varepsilon) \int_{r}^{R} \frac{1}{\rho} \int_{\rho}^{R} \oint_{S_{o}}|g r a d u|^{2} d s d \sigma d \rho
\end{aligned}
$$

for computable constant $\hat{C}$,

$$
\begin{aligned}
\left|\int_{r}^{R} \oint_{S_{\rho}} \rho p_{2} u u_{\rho} d s d \rho\right| \leqslant & M \oint_{S_{R}}\left|u u_{\rho}\right| d s+\frac{M^{2}}{2 R} \oint_{S_{R}} u^{2} d s \\
& +\int_{r}^{R} \oint_{S_{\rho}}\left(M p_{0}+\varepsilon\right) u^{2} d s d \rho \\
& +(M+\varepsilon) \int_{r}^{R} \oint_{S_{\rho}}|\operatorname{grad} u|^{2} d s d \rho \\
\left|\int_{r}^{R} \frac{1}{\rho} \int_{\rho}^{R} \oint_{S_{o}} \sigma p_{2} u u_{\sigma} d s d \sigma d \rho\right|< & \tilde{C} \ln \left(\frac{R}{r}\right) \oint_{S_{R}} u^{2} d s+M \ln \left(\frac{R}{r}\right) \oint_{S_{R}}|u|\left|u_{R}\right| d s \\
& +\int_{r}^{R} \frac{1}{\rho} \int_{\rho}^{R} \oint_{S_{o}}\left(M p_{0}+\varepsilon\right) u^{2} d s d \sigma d \rho \\
& +(M+\varepsilon) \int_{r}^{R} \frac{1}{\rho} \int_{\rho}^{R} \oint_{S_{o}}|\operatorname{grad} u|^{2} d s d \sigma d \rho(\mathrm{C} 4)
\end{aligned}
$$

for computable constant $\tilde{C}$.

We now make use of certain of these inequalities to establish the following lemma. 
LEMMA 2. Let $u$ be an $L_{2}$ solution of (1.1) and let $p$ satisfy the conditions (a)-(d) of the Main Theorem. Then

$$
\oint_{S_{r}}\left[|\operatorname{grad} u|^{2}-p_{0} u^{2}-2 u_{r}^{2}-2 C r^{-1} u u_{r}\right] d s \geqslant 0, \quad \forall r \geqslant R^{*},
$$

where $2 C=\gamma+n-2+2 M+\varepsilon$ for some positive $\gamma$, and where $\varepsilon>0$ may be made arbitrarily small by choosing $R^{*}$ sufficiently large.

In what follows we shall use $\varepsilon$ as a generic symbol to indicate a coefficient that may be made arbitrarily small by choosing $R^{*}$ sufficiently large. We shall not index the $\varepsilon$ 's except where it seems necessary for clarity.

Proof OF LEMMa 2. The proof of this lemma is based on a Rellich-type identity derived as follows:

Set

$$
\int_{r}^{R} \oint_{s_{\rho}}\left(\rho u_{\rho}+C u\right)(\Delta u+p u) d s d \rho=0, \quad r \geqslant R^{*}
$$

An application of divergence theorem gives

$$
\begin{aligned}
0 \equiv & \left.\frac{\rho}{2} \oint_{S_{\rho}}\left[2 u_{\rho}^{2}-|\operatorname{grad} u|^{2}+p_{0} u^{2}+\frac{2 C}{\rho} u u_{\rho}\right] d s\right|_{r} ^{R} \\
& +\frac{n-2-2 C}{2} \int_{r}^{R} \oint_{S_{\rho}}|\operatorname{grad} u|^{2} d s d \rho \\
& -\frac{1}{2} \int_{r}^{R} \oint_{S_{\rho}}\left[\rho \frac{\partial p_{0}}{\partial \rho}+(n-2 C) p_{0}\right] u^{2} d s d \rho \\
& +\int_{r}^{R} \oint_{S_{\rho}} \rho\left(p_{1}+p_{2}\right) u u_{\rho} d s d \rho+C \int_{r}^{R} \oint_{S_{\rho}}\left(p_{1}+p_{2}\right) u^{2} d s d \rho .
\end{aligned}
$$

We now make use of (B1), (B3), (C1) and (C3). Inserting the expression for $2 C$ into (1.6), choosing $\gamma=\hat{\gamma}$ (the $\hat{\gamma}$ of (d)) and rewriting (1.6), we obtain

$$
\begin{aligned}
J(R) \equiv & \frac{R}{2} \oint_{S_{R}}\left[2 u_{R}^{2}-|\operatorname{grad} u|^{2}+p_{0} u^{2}+\frac{2 C}{R} u u_{R}\right. \\
& \left.\quad+\frac{2 M}{R^{2}}(C+M) u^{2}\right] d s+M \oint_{S_{R}}\left|u u_{R}\right| d s \\
> & \frac{r}{2} \oint_{S_{r}}\left[2 u_{r}^{2}-|\operatorname{grad} u|^{2}+p_{0} u^{2}+\frac{2 C}{r} u u_{r}\right] d s \\
& +\left(\frac{\hat{\gamma}}{2}-\varepsilon\right) \int_{r}^{R} \oint_{S_{0}}\left[|\operatorname{grad} u|^{2}-u_{\rho}^{2}\right] d s d \rho \\
& +\frac{1}{2} \int_{r}^{R} \oint_{S_{\rho}}\left[\rho \frac{\partial p_{0}}{\partial \rho}+(2-4 M-\hat{\gamma}-\varepsilon) p_{0}-\frac{K^{2}}{\hat{\gamma}}-\varepsilon\right] u^{2} d s d \rho . \quad(1.7)
\end{aligned}
$$


Note that $J(R)$ satisfies

$$
\begin{aligned}
J(R)< & \frac{R}{2}\left\{\oint_{S_{R}}\left[|\operatorname{grad} u|^{2}+p_{0} u^{2}\right] d s\right\} \\
& +C_{4} \oint_{S_{r}}\left|u u_{R}\right| d s+\frac{C_{6}}{R} \int_{S_{R}} u^{2} d s
\end{aligned}
$$

and it is easily seen as indicated in [Ø], that

$$
\liminf _{R \rightarrow \infty} J(R)=0 \text {. }
$$

Thus, making use of the expression for $C$, using the fact that $u_{\rho}^{2}<|\operatorname{grad} u|^{2}$, inserting condition (d), and letting $R \rightarrow \infty$, we arrive at (1.5), and Lemma 2 is established.

We remark that it follows directly from (1.5) after integration and use of arithmetic-geometric mean inequality on the last term that

$$
\begin{aligned}
& \int_{r}^{R} \frac{1}{\rho} \int_{\rho}^{\infty} \oint_{S_{\sigma}} u_{\sigma}^{2} d s d \sigma d \rho \\
& \quad<\frac{1+\varepsilon}{2} \int_{r}^{R} \frac{1}{\rho} \int_{\rho}^{\infty} \oint_{S_{o}}\left[|\operatorname{grad} u|^{2}-\left(p_{0}-\varepsilon^{2}\right) u^{2}\right] d s d \sigma d \rho
\end{aligned}
$$

where again $r$ has been chosen sufficiently large. We shall make use of (1.9) later on.

Finally, we note that the integral

$$
\int_{r}^{\infty} \frac{1}{\rho} \int_{\rho}^{\infty} \oint_{S_{\sigma}} u^{2} d s d \sigma d \rho
$$

is convergent. This follows from the proof of Lemma 2 and hypothesis (d) of the Main Theorem. We have, from (1.7) after letting $R \rightarrow+\infty$ and using (1.8),

$$
\begin{aligned}
\oint_{S_{r}}\left[|\operatorname{grad} u|^{2}\right. & \left.-2 u_{r}^{2}-p_{0} u^{2}-\frac{2 C}{r} u u_{r}\right] d r \\
> & r^{-1}\left(\frac{\hat{\gamma}}{2}-\varepsilon\right) \int_{r}^{\infty} \oint_{S_{\rho}}\left[|\operatorname{grad} u|^{2}-u_{\rho}^{2}\right] d s d \rho \\
& +\frac{\hat{\varepsilon}}{r} \int_{r}^{\infty} \oint_{S_{\rho}} u^{2} d s d \rho,
\end{aligned}
$$

where $\varepsilon, \hat{\varepsilon}>0$ and $\varepsilon<\hat{\gamma} / 2$. Since $u, \nabla u \in L^{2}$ and $p_{0}$ is bounded, it follows from the above after a quadrature that the integral is convergent.

We shall need one additional lemma in the proof of the theorem, namely: 
LEMMA 3. Let $u$ be an $L_{2}$ solution of (1.1), and let $p$ satisfy conditions (a)-(d) of the theorem, then

$$
\begin{aligned}
& \int_{r}^{R} \frac{1}{\rho} \int_{\rho}^{\infty} \oint_{S_{\sigma}}|\operatorname{grad} u|^{2} d s d \sigma d \rho \\
& \quad<\frac{1+\varepsilon}{2} \oint_{S_{r}} \frac{u^{2}}{r} d s+(1+\varepsilon) \int_{r}^{\infty} \frac{1}{\rho} \int_{\rho}^{\infty} \oint_{S_{\sigma}}\left(p_{0}+\varepsilon\right) u^{2} d s d \sigma d \rho
\end{aligned}
$$

PROOF. The proof of this lemma comes from a straightforward integration by parts and inequalities (B) and (C). Noting that the boundary integrals at infinity may be dropped, we write

$$
\begin{aligned}
& \oint_{S_{r}} \frac{u^{2}}{r} d s=-2 \int_{r}^{R} \oint_{S_{\rho}} \frac{u}{\rho} u_{\rho} d s-(n-2) \int_{r}^{R} \oint_{S_{\rho}} \frac{u^{2}}{\rho^{2}} d s d \rho+\oint_{S_{R}} \frac{u^{2}}{R} d s \\
& =2 \int_{r}^{R} \frac{1}{\rho} \int_{\rho}^{\infty} \frac{d}{d \sigma}\left\{\oint_{S_{\sigma}}\left[u u_{\sigma}+\frac{(n-2)}{2} \frac{u^{2}}{\sigma}\right] d s\right\} d \sigma d \rho+\oint_{S_{R}} \frac{u^{2}}{R} d s \\
& \quad=2 \int_{r}^{R} \frac{1}{\rho} \int_{\rho}^{\infty} \oint_{S_{\sigma}}\left[|\operatorname{grad} u|^{2}-p u^{2}+(n-2) \frac{u u_{\sigma}}{\sigma}\right. \\
& >(2-\varepsilon) \int_{r}^{R} \frac{1}{\rho} \int_{\rho}^{\infty} \oint_{S_{\sigma}}|\operatorname{grad} u|^{2} d s d \sigma d \rho \\
& \left.\sigma^{2}\right] d s d \sigma d \rho+\oint_{S_{R}} \frac{u^{2}}{R} d s \\
& \quad-\int_{r}^{R} \frac{1}{\rho} \int_{\rho}^{\infty} \oint_{S_{\sigma}}\left(2 p_{0}+\varepsilon\right) u^{2} d s d \sigma d \rho+\oint_{S_{R}} \frac{u^{2}}{R} d s .
\end{aligned}
$$

Solving for the first term on the right, we find, after letting $R \rightarrow+\infty$,

$$
\begin{aligned}
& \int_{r}^{\infty} \frac{1}{\rho} \int_{\rho}^{\infty} \oint_{S_{o}}|\operatorname{grad} u|^{2} d s d \sigma d \rho \\
& \quad \leqslant \frac{1}{2-\varepsilon} \oint_{S_{r}} \frac{u^{2}}{r} d s+\int_{r}^{\infty} \frac{1}{\rho} \int_{\rho}^{\infty} \oint_{S_{o}}\left(\frac{2 p_{0}+\varepsilon}{2-\varepsilon}\right) u^{2} d s d \sigma d \rho
\end{aligned}
$$

which is equivalent to (1.10). Note that both sides of this last inequality are finite.

Note. Inequalities (1.9) and (1.10) can be combined to give

$$
\begin{aligned}
& \int_{r}^{\infty} \frac{1}{\rho} \int_{\rho}^{\infty} \oint_{S_{\sigma}} u_{\sigma}^{2} d s d \sigma d \rho \\
& \quad<\left(\frac{1+\varepsilon}{2}\right)^{2} \oint_{S_{r}} \frac{u^{2}}{r} d s+\varepsilon \int_{r}^{\infty} \frac{1}{\rho} \int_{\rho}^{\infty} \oint_{S_{\sigma}}\left(p_{0}+\varepsilon\right) u^{2} d s d \sigma d \rho
\end{aligned}
$$


We are now ready to compute a lower bound for $I_{1}$ in terms of $F$ and $F^{\prime}$, and complete the proof of the Main Theorem.

Proof of the Mann Theorem. In (1.6) take lim inf as $R \rightarrow \infty$ and set $C=0$. (Note that the special choice for $C$ used in proving Lemma 2 has not been made at this point.) Then dividing by $\rho$ and integrating, we get

$$
\begin{aligned}
\frac{1}{2} I_{1} \geqslant & -\frac{n-2}{2} \int_{r}^{\infty} \frac{1}{\rho} \int_{\rho}^{\infty} \oint_{S_{\sigma}}|\operatorname{grad} u|^{2} d s d \sigma d \rho \\
& -\frac{1}{2} \int_{r}^{\infty} \frac{1}{\rho} \int_{\rho}^{\infty} \oint_{S_{\sigma}}\left[\sigma \frac{\partial p_{0}}{\partial \sigma}+n p_{0}\right] u^{2} d s d \sigma d \rho \\
& +\int_{r}^{\infty} \frac{1}{\rho} \int_{\rho}^{\infty} \oint_{S_{\sigma}} \sigma\left(p_{1}+p_{2}\right) u u_{\sigma} d s d \sigma d \rho \\
& -\frac{1}{2} \int_{r}^{\infty} \oint_{S_{\rho}}\left(p_{1}+p_{2}\right) u^{2} d s d \rho .
\end{aligned}
$$

We now apply (B2), (B4), (C2) and (C4), after letting $R \rightarrow \infty$, we obtain, for some positive $\gamma$,

$$
\begin{aligned}
\frac{1}{2} I_{1}> & -\frac{1}{2}(n-2+4 M+\varepsilon) \int_{r}^{\infty} \frac{1}{\rho} \int_{\rho}^{\infty} \oint_{S_{o}}|\operatorname{grad} u|^{2} d s d \sigma d \rho \\
& -\gamma \int_{r}^{\infty} \frac{1}{\rho} \int_{\rho}^{\infty} \oint_{S_{o}} u_{o}^{2} d s d \sigma d \rho \\
& +\frac{1}{2} \int_{r}^{\infty} \frac{1}{\rho} \int_{\rho}^{\infty} \oint_{S_{o}}\left[\sigma \frac{\partial p_{0}}{\partial \sigma}+(n-4 M) p_{0}-\frac{2 K^{2}}{\gamma}-\varepsilon\right] u^{2} d s d \sigma d \rho
\end{aligned}
$$

Using (1.10) and (1.11), we obtain

$$
\begin{aligned}
\frac{1}{2} I_{1}> & -\frac{1+\varepsilon}{4}(n-2+4 M+\varepsilon+\gamma) \oint_{S_{r}} \frac{u^{2}}{r} d s \\
& +\hat{\varepsilon} \int_{r}^{\infty} \frac{1}{\rho} \int_{\rho}^{\infty} \oint_{S_{o}} u^{2} d s d \sigma d \rho \\
& +\frac{1}{2} \int_{r}^{\infty} \frac{1}{\rho} \int_{\rho}^{\infty} \oint_{S_{\sigma}}\left[\sigma \frac{\partial p_{0}}{\partial \sigma}+2(1-4 M+\varepsilon) p_{0}-\frac{2 K^{2}}{\gamma}-\varepsilon\right] u^{2} d s d \sigma d \rho
\end{aligned}
$$

Let us now assume that $\gamma$ is such that at each point of $S_{r}$

$$
r \frac{\partial p_{0}}{\partial r}+2(1-4 M-\varepsilon) p_{0}-\frac{2 K^{2}}{\gamma}-\varepsilon \geqslant 0
$$


holds. Then (1.14) may be rewritten as

$$
\begin{aligned}
\frac{1}{2} I_{1} \geqslant & -\frac{(1+\varepsilon)(n-2)}{4}(n-2+4 M+\varepsilon+\gamma) t^{-1} F^{\prime}(t) \\
& +\frac{\hat{\varepsilon}}{n-2} \int_{0}^{t} \eta^{-(3 n-4) /(n-2)} F(\eta) d \eta
\end{aligned}
$$

since clearly

$$
\int_{r}^{\infty} \frac{1}{\rho} \int_{\rho}^{\infty} \oint_{S_{\sigma}} u^{2} d s d \sigma d \rho \geqslant \int_{r}^{\infty} \rho^{2 n-3} F(\rho) d \rho
$$

In order to apply Lemma 1 , and conclude that $u \equiv 0$ in $B^{c}\left(R^{*}\right)$, we must require that $\gamma$ satisfy the inequality

$$
2 \frac{(1+\varepsilon)(n-2+4 M+\varepsilon+\gamma)}{n-2}+1<\frac{3 n-4}{n-2},
$$

i.e., we may choose

$$
\gamma=1-4 M-\varepsilon_{1} .
$$

Thus, if (1.15) is satisfied with $\gamma$ given by (1.16), we conclude from Lemma 1 that $u \equiv 0$ in $B^{c}\left(R^{*}\right)$. But the insertion of (1.16) into (1.15) yields the assumed condition (e) and the theorem is proved. Q.E.D.

II. Examples and remarks. (1) A class of unbounded and oscillatory potentials which have not been considered by previous authors are those of following type:

$$
V(r)=A r^{\delta} \sin r^{\beta}
$$

If we let $V_{2}(x)=V(r)$, then

$$
\left|A \int_{r}^{\rho} \sigma^{\delta+1} \sin \sigma^{\beta} d \sigma\right| \leqslant \frac{2 A}{\beta} \frac{1}{r^{\beta-2-\delta}} .
$$

If we now choose $\delta+2<\beta$ than the right-hand side, for fixed $A$ and $\beta$, will become arbitrarily small for $r>R^{*}$ (sufficiently large). By Theorem 4, $-\Delta+V$ possesses no eigenvalue $\lambda \in(0, \infty)$.

(2) Let $V(r)=H(r) / r$ where $H$ is defined for $l, h>0$ as

$$
H(r)= \begin{cases}h: & 2 j l \leqslant r<(2 j+1) l \\ -h: & (2 j+1) l \leqslant r<2(j+1) l, \quad j=0,1, \ldots\end{cases}
$$

If $h l<\frac{1}{4}$, and if we set $p=p_{2}=\lambda-V$, it follows as a consequence of the Main Theorem that there exists in $\Omega$ no nontrivial $L_{2}$ solution of (1.1). If $h l>\frac{1}{4}$, and if we set $p=p_{1}=\lambda-V$, it follows from the Main Theorem that no nontrivial $L_{2}$ solution for equation (1.1) will exist for $\lambda>h^{2}$.

(3) For a class of potentials which contains that of von Neumann and Wigner, namely those potentials of the form 


$$
V= \pm \frac{A \sin B r}{r}
$$

(oscillatory and of order $1 / r$ at infinity), Theorem 4 provides in many cases an improved bound for the largest eigenvalue, the improvement depending upon the relative sizes of $A$ and $B$. For example, if we assume $V(x) \equiv V_{2}(x)$ $= \pm(A \sin B r) / r$, then

$$
\left|A \int_{r}^{\rho} \sigma \frac{\sin B \sigma}{\sigma} d \sigma\right|=\left|\frac{A}{B} \cos B \sigma\right|_{r}^{\rho} \mid \leqslant \frac{2 A}{B} .
$$

Hence, for $2 A / B<\frac{1}{4}$ no eigenvalue exists in $(0, \infty)$. On the other hand, if we let $V(r)=V_{1}(r)= \pm(A \sin B r) / r$, then from Theorem 4 we conclude that $\alpha=A^{2}$, and hence there exists no eigenvalue $\lambda \in\left(A^{2}, \infty\right)$. If we define $V(r)=V_{0}(r)= \pm(A \sin B r) / r$, then

$$
\Lambda_{0}=\limsup _{r \rightarrow \infty}\left( \pm r \frac{A B r \cos B r-A \sin B r}{r^{2}}\right)=A B \text {. }
$$

By Theorem 4, again we conclude that no eigenvalues for the operator $-\Delta \pm(A \sin B r) / r$ exists in the interval $(A B / 2,+\infty)$. Therefore, if $2 A / B<\frac{1}{4}$ we take $V=V_{2}$; otherwise we choose $V=V_{0}$ or $V=V_{1}\left(V_{1}\right.$ in case $\frac{1}{4} \leqslant 2 A / B<1$, and $V_{0}$ if $\left.2 A / B>1\right)^{3}$

We remark that the nature of the spectrum in the interval $(0, \alpha)$ for the class of long range potentials considered in this paper remains unknown. The above examples illustrate that the value of $\alpha$ frequently depends on the way in which $p(x)$ is decomposed. In a specific example one will choose the optimal decomposition if it can be determined.

Appendix. We present here the proofs of the inequalities (B) and (C).

$$
\begin{aligned}
& \left|\int_{r}^{R} \oint_{S_{\rho}} p_{1} u^{2} d s d \rho\right| \leqslant \frac{K}{r} \int_{r}^{R} \oint_{S_{\rho}} u^{2} d s d \rho=\varepsilon \int_{r}^{R} \oint_{S_{\rho}} u^{2} d s d \rho \\
& \left|\int_{r}^{R} \oint_{S_{\rho}} p_{1} u^{2} d s d \rho\right| \leqslant K \int_{r}^{R} \frac{1}{\rho} \oint_{S_{\rho}} u^{2} d s d \rho \\
& =K \int_{r}^{R} \frac{1}{\rho}\left\{\oint_{S_{R}} u^{2} d s-\int_{\rho}^{R} \oint_{S_{o}}\left[2 u u_{\sigma}+\frac{n-1}{\sigma} u^{2}\right] d s d \sigma\right\} d \rho \\
& \leqslant K \ln \left(\frac{R}{r}\right) \oint_{S_{R}} u^{2} d s+\int_{r}^{R} \frac{1}{\rho} \int_{\rho}^{R} \oint_{S_{o}}\left[\gamma u_{\sigma}^{2}+\frac{K^{2}}{\gamma} u^{2}\right] d s d \sigma d \rho ;
\end{aligned}
$$

\footnotetext{
${ }^{3}$ The authors are indebted to $B$. Simon for drawing their attention to several papers dealing with oscillatory potentials. (See e.g. F. V. Atkinson, Ann. Math. Pura Appl. 37 (1954), 347-378; S. Albeverio, Ann. Phys. 71 (1972), 167-276, and papers cited in Reed and Simon, Modern methods of mathematical physics, Vol. III, IV.) They also wish to thank the referee for a number of helpful suggestions.
} 


$$
\begin{aligned}
\left|\int_{r}^{R} \oint_{S_{\rho}} \rho p_{1} u u_{\rho} d s d \rho\right| & \leqslant K \int_{r}^{R} \oint_{S_{\rho}}\left|u u_{\rho}\right| d s d \rho \\
& \leqslant \frac{1}{2} \int_{r}^{R} \oint_{S_{\rho}}\left[\gamma u_{\rho}^{2}+\frac{K^{2}}{\gamma} u^{2}\right] d s d \rho .
\end{aligned}
$$

$$
\begin{aligned}
\left|\int_{r}^{R} \oint_{S_{\rho}} p_{2} u^{2} d s d \rho\right| & =\left|\int_{r}^{R} \oint_{S_{\rho}} \frac{\partial g(\rho, r)}{\partial \rho} \frac{u^{2}}{\rho} d s d \rho\right| \\
& =\left|\oint_{S_{R}} \frac{g(R, r)}{R} u^{2} d s-\int_{r}^{R} \oint_{S_{\rho}} \frac{g}{\rho}\left[2 u u_{\rho}+(n-2) \frac{u^{2}}{\rho}\right] d s d \rho\right| \\
& <\frac{M}{R} \oint_{S_{R}} u^{2} d s+\varepsilon \int_{r}^{R} \oint_{S_{\rho}}\left[u_{\rho}^{2}+u^{2}\right] d s d \rho .
\end{aligned}
$$

We have introduced the notation $g(\rho, r)=\int_{r}^{\rho} \sigma p_{2}(\sigma \omega) d \sigma$, dropping the indication of dependence on $\omega$.

$$
\begin{aligned}
J_{0} & =\left|\int_{r}^{R} \oint_{S_{\rho}} p_{2} u^{2} d s d \rho\right|=\left|\int_{r}^{R} \oint_{S_{\rho}} \frac{\partial}{\partial \rho} g(s, r) \frac{u^{2}}{\rho} d s d \rho\right| \\
& =\left|\oint_{S_{R}} g(R, r) \frac{(u(R))^{2}}{R} d s-\int_{r}^{R} \oint_{S_{\rho}} g(s, r)\left[\frac{2 u u_{\rho}}{\rho}+\frac{(n-2)}{\rho^{2}} u^{2}\right] d s d \rho\right| \\
& <\frac{M}{R} \oint_{S_{R}} u^{2} d s+\left|\int_{r}^{R} \oint_{S_{\rho}} \frac{\partial}{\partial \rho} h(\rho, r)\left[2 u u_{\rho}+\frac{n-2}{\rho} u^{2}\right] d s d \rho\right|
\end{aligned}
$$

We let

$$
J_{1}=\frac{M}{R} \oint_{S_{R}} u^{2} d s, \quad h(\rho, r)=\int_{r}^{\rho} \frac{g(\sigma, r)}{\sigma} d \sigma
$$

and note that $|h|<M \ln (R / r)$. Then

$$
\begin{aligned}
J_{0}< & J_{1}+\left|\oint_{S_{R}} h(R, r)\left[2 u u_{R}+\frac{n-2}{R} u^{2}\right] d s\right| \\
& +2\left|\int_{r}^{R} \oint_{S_{\rho}} h(\rho, r)\left[|\operatorname{grad} u|^{2}-p u^{2}+\frac{n-2}{\rho} u u_{\rho}+\frac{(n-2)^{2}}{2 \rho^{2}} u^{2}\right] d s d \rho\right| .
\end{aligned}
$$

For simplicity, we denote by $J_{2}$ the second integral on the right-hand side. Thus, we obtain 


$$
\begin{gathered}
J_{0}<J_{1}+2 M \ln \left(\frac{R}{r}\right) \oint_{S_{R}}\left|u u_{R}\right| d s+\frac{M(n-2)}{R} \ln \left(\frac{R}{r}\right) \oint_{S_{R}} u^{2} d s+2 J_{2} . \\
J_{2}<\left|\int_{r}^{R} \oint_{S_{\rho}} h(\rho, r)\left[|\operatorname{grad} u|^{2}-p_{0} u^{2}\right] d s d \rho\right|+\left|\int_{r}^{R} \oint_{S_{\rho}} h(\rho, r) p_{2} u^{2} d s d \rho\right| \\
\left.+\left|\int_{r}^{R} \oint_{S_{\rho}} h(\rho, r)\right| \frac{n-2}{\rho} u u_{\rho}-p_{1} u^{2}+\frac{(n-2)^{2}}{2 \rho^{2}} u^{2}\right] d s d \rho \mid \\
\equiv J_{21}+J_{22}+J_{23} . \\
J_{21}<M \int_{r}^{R} \int_{r}^{\rho} \frac{d \sigma}{\sigma} \oint_{S_{\rho}}\left[|\operatorname{grad} u|^{2}+p_{0} u^{2}\right] d s d \rho \\
\quad<M \int_{r}^{R} \frac{1}{\rho} \int_{\rho}^{R} \oint_{S_{0}}\left[|\operatorname{grad} u|^{2}+p_{0} u^{2}\right] d s d \sigma d \rho, \\
J_{22} \equiv\left|\int_{r}^{R} \oint_{S_{\rho}}\left(\int_{r}^{\rho} \frac{g(\sigma, r)}{\sigma} d \sigma\right) p_{2} u^{2} d s d \rho\right| \\
=\left|\int_{r}^{R} \int_{r}^{\rho} \oint_{|w|=1} \frac{g(\sigma, r)}{\sigma} \frac{\partial}{\partial \rho} g(\rho, \sigma) u^{2} \rho^{n-2} d w d \sigma d \rho\right| .
\end{gathered}
$$

After interchanging the order of $\sigma, \rho$ integration and performing an integration by parts, we find that

$$
\begin{aligned}
& J_{22}=\mid \int_{r}^{R} \oint_{S_{R}} \frac{g(\sigma, r)}{\sigma} \frac{g(R, \sigma)}{R} u^{2} d s d \sigma \\
&-\int_{r}^{R} \int_{0}^{R} \oint_{S_{\rho}} \frac{g(\sigma, r) g(\rho, r)}{\rho \sigma}\left[2 u u_{\rho}+\frac{\eta-2}{\rho} u^{2}\right] d s d \rho d \sigma \mid
\end{aligned}
$$

Using the assumed bound $|g(\sigma, r)|<M$, we obtain

$$
\begin{aligned}
& J_{22}<\frac{M^{2}}{R} \ln (R / r) \oint_{S_{R}} u^{2} d s+\varepsilon \int_{r}^{R} \frac{1}{\sigma} \int_{r}^{R} \oint_{S_{\rho}}\left[u^{2}+u_{\rho}^{2}\right] d s d \rho d \sigma \\
& J_{23}=\int_{r}^{R} \oint_{S_{\rho}} h(\rho, r)\left[\frac{n-2}{\rho} u_{\rho} u+\frac{\rho p_{1}}{\rho} u^{2}+\frac{(n-2)^{2}}{\rho^{2}} u^{2}\right] d s d \rho \\
& <M \int_{r}^{R} \oint_{S_{\rho}} \int_{r}^{\rho} \frac{d \sigma}{\sigma}\left[\frac{(n-2)}{\rho}\left|u_{\rho} u\right|+\frac{K}{\rho} u^{2}+\frac{(n-2)^{2}}{\rho^{2}} u^{2}\right] d s d \rho \\
& <\frac{M}{r} \int_{r}^{R} \frac{1}{\sigma} \int_{\sigma}^{R} \oint_{S_{\rho}}\left[\frac{n-2}{2}\left(u^{2}+u_{\rho}^{2}\right)+K u^{2}+\frac{(n-2)^{2}}{\rho} u^{2}\right] d s d \rho \\
& <M \varepsilon \int_{r}^{R} \frac{1}{\rho} \int_{\rho}^{R} \oint_{S_{\sigma}}\left(u^{2}+u_{\rho}^{2}\right) d s d \sigma d \rho .
\end{aligned}
$$


Combining all the above inequalities we arrive at (C2), i.e.,

$$
\begin{aligned}
& J_{0}=\left|\int_{r}^{R} \oint_{S_{\rho}} p_{2} u^{2} d s d \rho\right| \leqslant \varepsilon \oint_{S_{R}} u^{2} d s+\tilde{C} \ln (R / r) \oint_{S_{R}}\left|u u_{R}\right| d s \\
&+\int_{r}^{R} \frac{1}{\rho} \int_{\rho}^{R} \oint_{S_{o}}\left(2 M p_{0}+\varepsilon\right) u^{2} d s d \sigma d \rho \\
&+(2 M+\varepsilon) \int_{r}^{R} \frac{1}{\rho} \int_{\rho}^{R} \oint_{S_{\sigma}}|\operatorname{grad} u|^{2} d s d \sigma d \rho ; \\
&\left|\int_{r}^{R} \oint_{S_{\rho}} \rho p_{2} u u_{\rho} d s d \rho\right|=\left|\int_{r}^{R} \oint_{S_{\rho}} \frac{\partial g(\rho, r)}{\partial \rho} u u_{\rho} d s d \rho\right| \\
&=\mid \oint_{S_{R}} g(R, r) u u_{R} d s-\int_{r}^{R} \oint_{S_{\rho}} g(\rho, r)\left[|\operatorname{grad} u|^{2}-p_{0} u^{2}\right] d s d \rho \\
&+\int_{r}^{R} \oint_{S_{\rho}}\left(p_{1}+p_{2}\right) g(\rho, r) u^{2} d s d \rho \mid \\
& \leqslant M \oint_{S_{R}}|u|\left|u_{R}\right| d s+M \int_{r}^{R} \oint_{S_{\rho}}\left[|\operatorname{grad} u|^{2}+p_{0} u^{2}\right] d s d \rho \\
&+\left|\int_{r}^{R} \oint_{S_{\rho}} g(\rho, r)\left(p_{1}+p_{2}\right) u^{2} d s d \rho\right| .
\end{aligned}
$$

The last integral is then split into the sum of two integrals, the first of which is treated using (B1) and the second of which can be written as

$$
\left|\int_{r}^{R} \oint_{S_{\rho}} g(\rho, r) p_{2} u^{2} d s d \rho\right|=\frac{1}{2}\left|\int_{r}^{R} \oint_{S_{\rho}} \frac{\partial}{\partial \rho} g^{2}(\rho, r) \cdot \frac{u^{2}}{\rho} d s d \rho\right| ;
$$

An integration by parts then leads to (C3).

$$
\begin{aligned}
\left|\int_{r}^{R} \frac{1}{\rho} \int_{\rho}^{R} \oint_{S_{o}} \sigma p_{2} u u_{\sigma} d s d \sigma d \rho\right|=\left|\int_{r}^{R} \frac{1}{\rho} \int_{\rho}^{R} \oint_{S_{o}} \frac{\partial g(\sigma, \rho)}{\partial \sigma} u u_{\sigma} d s d \sigma d \rho\right| \\
<\left|\int_{r}^{R} \frac{1}{\rho} \oint_{S_{R}} g(R, \rho) u u_{R} d s d \rho\right| \\
\quad+\left|\int_{r}^{R} \frac{1}{\rho} \int_{\rho}^{R} \oint_{S_{o}} g(\sigma, \rho)\left[|\operatorname{grad} u|^{2}-p u^{2}\right] d s d \sigma d \rho\right| \\
<M \ln \left(\frac{R}{r}\right) \oint_{S_{R}}\left|u u_{R}\right| d s \\
+M \int_{r}^{R} \frac{1}{\rho} \int_{\rho}^{R} \oint_{S_{o}}\left[|\operatorname{grad} u|^{2}+p_{0} u^{2}\right] d s d \sigma d \rho \\
+\left|\int_{r}^{R} \frac{1}{\rho} \int_{\rho}^{R} \oint_{S_{o}} g(\sigma, \rho)\left(p_{1}+p_{2}\right) u^{2} d s d \sigma d \rho\right|
\end{aligned}
$$


and the proof is again completed by use of (B1) and the argument for (C3). Q.E.D.

NOTE ADDED IN PROOF. A special case of Theorem 4 has its analog in the case of one space dimension. If $M=0$, then the content of Theorem 4 is exactly the same as a result of Eastham (Lecture Notes in Math. 564 (1976), pp. 72-77, Springer-Verlag, Berlin and New York). On the other hand, it is not known that if (*) is replaced by

$$
\lim _{r \rightarrow+\infty} \sup r\left|\partial V_{0} / \partial r\right|=\Lambda_{0}
$$

and $M=0$, then the improved result of Knowles holds (Proc. Roy. Soc. Edinburgh 80A (1978), 15-22) namely $H$ has no eigenvalues in $(\alpha,+\infty)$ where $\alpha=\frac{1}{2}\left(K^{2}+\left(K^{4}+\Lambda_{0}^{2}\right)^{1 / 2}\right)$.

\section{REFERENCES}

1. S. Agmon, Lower bounds for solutions of Schrödinger equations, J. Analyse Math. 23 (1970), $1-25$.

2. L Lower bounds for solutions of Schrödinger-type equations in unbounded domains, Proc. Internat. Conf. Functional Analysis and Related Topics, Tokyo, 1969, pp. 216-224. 1963.

3. L. Hörmander, Linear partial differential operators, Springer-Verlag, Berlin and New York,

4. T. Kato, Growth properties of the reduced wave equation with variable coefficients, Comm. Pure Appl. Math. 12 (1959), 403-425.

5. Some mathematical problems in quantum mechanics, Progr. Theoret. Phys. 40 (1967), 3-14.

6. G. B. Khosrovshahi, Nonexistence of nontrivial solutions of Schrödinger type systems, SIAM J. Math. Anal. 8 (1977), 998-1013.

7. R. Konno, Non-existence of pasitive eigenvalues of Schrödinger operators in infinite domains, J. Fac. Sci. Univ. Tokyo Sect. I A Math. 19 (1972), 393-402.

8. R. Lavine, Absolute continuity of positive spectrum for Schrödinger operators with long range potentials, J. Functional Analysis 12 (1973), 30-54.

9. F. Odeh, Note on differential operators with purely continuous spectrum, Proc. Amer. Math. Soc. 16 (1965), 363-366.

10. B. Simon, On positive eigenvalues of one-body Schrödinger operators, Comm. Pure Appl. Math. 22 (1969), 531-538.

11. J. von Neumann and E. P. Wigner, Über merkwürdige diskrete Eigenwerte, Phys. Z. 50 (1929), 465-467.

12. J. Weidmann, On the continuous spectrum of Schrödinger type operators, Comm. Pure Appl. Math. 19 (1966), 107-110.

13. Bull. Amer. Math. Soc. 73 (1967), 452-459.

Department of Mathematics, University of Tehran, Tehran, Iran

Department of Mathematics, Iowa State UntverstTy, Ames, Iowa 50011

Department of Mathematics, Cornell Untverstry, IthacA, New York 14853 (Current address of G. B. Khosrovshahi and L. E. Payne)

Current address (H. A. Levine): Department of Mathematics, Iowa State University, Ames, Iowa 50011 